\title{
G17-modified hammerhead ribozymes are active in vitro and in vivo
}

\author{
ANNE KALWEIT and CHRISTIAN HAMMANN ${ }^{1}$ \\ Ribogenetics@Biochemistry Lab, School of Engineering and Science, MoLife Research Center, Jacobs University Bremen, 28759 Bremen, Germany
}

\begin{abstract}
Natural hammerhead ribozymes (HHRz) feature tertiary interactions between hairpin loops or bulges in two of three helices that surround the catalytic core of conserved nucleotides. Their conservation was originally established on minimal versions lacking the tertiary interactions. While those sequence requirements in general also apply to natural versions, we show here differences for the HHRz cleavage site N17. A guanosine at this position strongly impairs cleavage activity in minimal versions, whereas we observe for the G17 variants of four tertiary stabilized HHRz significant cleavage and ligation activity in vitro. Kinetic analyses of these variants revealed a reduced rate and extent of cleavage, compared with wild-type sequences, while variants with distorted tertiary interactions cleaved at a reduced rate, but to the same extent. Contrary to this, G17 variants exhibit similar in vitro ligation activity as compared with the respective wild-type motif. To also address the catalytic performance of these motifs in vivo, we have inserted HHRz cassettes in the lacZ gene and tested this $\beta$-galactosidase reporter in Dictyostelium discoideum. In colorimetric assays, we observe differences in the enzymatic activity of $\beta$-galactosidase, which correlate well with the activity of the different HHRz variants in vitro and which can be unambiguously attributed to ribozyme cleavage by primer extension analysis.
\end{abstract}

Keywords: catalytic RNA; self-cleavage; ligation; Dictyostelium discoideum; tertiary interactions

\section{INTRODUCTION}

The hammerhead ribozyme (HHRz) is a small RNA endonuclease composed of a conserved catalytic core and three surrounding helical stems (Fig. 1; Uhlenbeck 1987; Hammann and Lilley 2002). In natural HHRz, the capping loops two of these helices, HI and HII, engage in tertiary interactions, leading to accelerated cleavage and ligation (De la Peña et al. 2003; Khvorova et al. 2003) and accelerated folding (Penedo et al. 2004) under physiological $\mathrm{Mg}^{2+}$ concentrations. If these tertiary interactions are disabled, the resulting minimal $\mathrm{HHRz}$ will still cleave, however, only at elevated $\mathrm{Mg}^{2+}$ concentrations above $10 \mathrm{mM}$. Such minimal versions were used to determine the core nucleotide requirements by extensive kinetic analyses (Ruffner et al. 1990), resulting in the HHRz consensus core sequence depicted in Figure 1A. From detailed in vitro analyses (Uhlenbeck 1987; Koizumi and Ohtsuka 1991; Slim and Gait 1991; van Tol et al. 1991), an $S_{N} 2$ mechanism (Fig. 1B) was proposed to be operational in the HHRz catalysis. In the presence of tertiary interactions, HHRzs have a higher propensity to undergo a reverse ligation reaction than in minimal forms (De la Peña et al. 2003; Khvorova et al. 2003).

\footnotetext{
${ }^{1}$ Corresponding author

E-mail c.hammann@jacobs-university.de

Article published online ahead of print. Article and publication date are at http://www.rnajournal.org/cgi/doi/10.1261/rna.040543.113.
}

Several studies have indicated a great variation of tertiary interacting sequences in individual HHRz motifs (Przybilski and Hammann 2007a; Shepotinovskaya and Uhlenbeck 2008), although recurrent signatures were also observed (Dufour et al. 2009). The variability of tertiary interacting motifs became even further apparent in several recent database searches for novel $\mathrm{HHRz}$ motifs carried out in this and other laboratories (Ferbeyre et al. 2000; Rojas et al. 2000; Gräf et al. 2005; Przybilski et al. 2005; Martick et al. 2008; De la Peña and Garcia-Robles 2010; Jimenez et al. 2011; Perreault et al. 2011; Seehafer et al. 2011). While the HHRz was originally discovered in subviral plant pathogens (Hutchins et al. 1986; Prody et al. 1986; Hammann and Steger 2012), the aforementioned searches have revealed many, predominantly eukaryotic species, with genomically encoded HHRz motifs (for recent review, see Hammann et al. 2012).

Extensive crystallographic analyses by Scott and coworkers have revealed that the presence of tertiary interacting elements also leads to a dramatic change in the conformation of the catalytic core (Martick and Scott 2006), in particular by the formation of a Watson-Crick base pair between

(C) 2013 Kalweit and Hammann This article is distributed exclusively by the RNA Society for the first 12 months after the full-issue publication date (see http://rnajournal.cshlp.org/site/misc/terms.xhtml). After 12 months, it is available under a Creative Commons License (Attribution-NonCommercial 3.0 Unported), as described at http://creativecommons.org/licenses/by-nc/ $3.0 /$. 

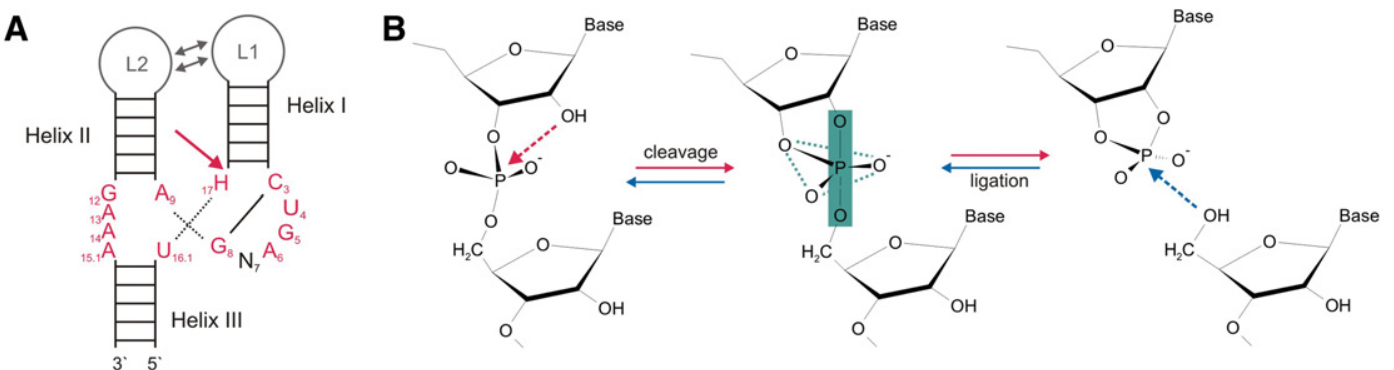

FIGURE 1. The hammerhead ribozyme (HHRz). (A) Schematic representation of the secondary structure. The conserved nucleotides of the catalytic core are shown in red using the conventional numbering system (Hertel et al. 1992). The surrounding helices I, II, and III are depicted together with the interacting loops L1 and L2. (Solid lines) Watson-Crick base-pairing; (dashed lines) backbone continuities. (Arrow) The cleavage site. (B) The reaction mechanism of the HHRz. Self-cleavage (red arrows) is initiated by a nucleophilic attack of the $2^{\prime}$-hydroxyl on the neighboring $3^{\prime}, 5^{\prime}$-phosphodiester and proceeds via a penta-coordinated transition state with inline geometry (green). That is resolved to generate a $5^{\prime}$ product with a $2^{\prime}, 3^{\prime}$-cyclic phosphate and a $3^{\prime}$ product with a $5^{\prime}$ hydroxyl. The reverse ligation reaction (blue arrows) in turn is initiated by a nucleophilic attack of the $5^{\prime}$ hydroxyl on the cyclic phosphate.

nucleotides C3 and G8 (Fig. 1). That base pair was later also confirmed in two independent studies (Przybilski and Hammann 2007b; Nelson and Uhlenbeck 2008b). It had not, however, been observed in crystal structures of minimal HHRz versions (Murray et al. 1998), which appear to represent a ground state of the ribozyme, despite the fact that cleavage was observed in crystals (Scott et al. 1996). This finding and discrepant biochemical data led to a substantial discussion on the mechanism of the HHRz reaction (Wang et al. 1999; Murray and Scott 2000; Blount and Uhlenbeck 2005). The first crystal structure of a tertiary stabilized HHRz (Martick and Scott 2006) helped to reconcile the opposing positions that had dominated the field for some time, as summarized in Przybilski and Hammann (2006). Subsequently, a study using adiabatic morphing calculations indicated that the two structures can be interconverted (Scott 2007). Also, the biochemical data on the core nucleotide requirements, inferred with minimal versions (Ruffner et al. 1990), were shown to be in general consistent with the structure of the tertiary stabilized HHRz (Nelson and Uhlenbeck 2008a). Both these studies implied that minimal HHRz exhibit a reduced reaction rate because they first need to undergo a structural change before reaching the transition state. Once this happens, however, both types of HHRz are thought to use the same chemical mechanism for the cleavage reaction. In this, the tertiary interacting elements in $\mathrm{HI}$ and $\mathrm{HII}$ serve mainly to speed up the reaction, in line with their established function as auxiliary elements in folding (Penedo et al. 2004).

One of the most conserved positions in the HHRz core is $\mathrm{H} 17$, as documented in a high number of studies (Ruffner et al. 1990; Shimayama et al. 1995; Zoumadakis and Tabler 1995; Baidya and Uhlenbeck 1997; Baidya et al. 1997; Kore et al. 1998, 2000; Przybilski and Hammann 2007b; Nelson and Uhlenbeck 2008b; Shepotinovskaya and Uhlenbeck 2010), leading to the establishment of the NUH rule for the HHRz cleavage site. As reason for the dramatically reduced functionality of HHRzs with a guanosine at position 17, the formation of a Watson-Crick base pair with C3 was pre- sumed (Fig. 2A), which would be stabilized by stacking onto helix I. In view of the unavailability of C3 in tertiary stabilized HHRz for this interaction, we wondered whether the NUH rule holds also in this case. This was also furthered by a surprisingly high activity of a G17-containing HHRz that we encountered during the characterization of a subset of $\mathrm{HHRz}$ motifs identified recently (Seehafer et al. 2011). Using cleavage and ligation analyses, an HHRz-coupled $\beta$-galactosidase assay, and primer extension reactions, we show here that G17 variants are active in vitro and in vivo in all four tested tertiary stabilized HHRz motifs.

\section{RESULTS}

\section{Substantial cleavage activity of hammerhead ribozymes with a G17 cleavage site}

Using different pattern descriptors and search algorithms, the various recent database searches identified a large number of

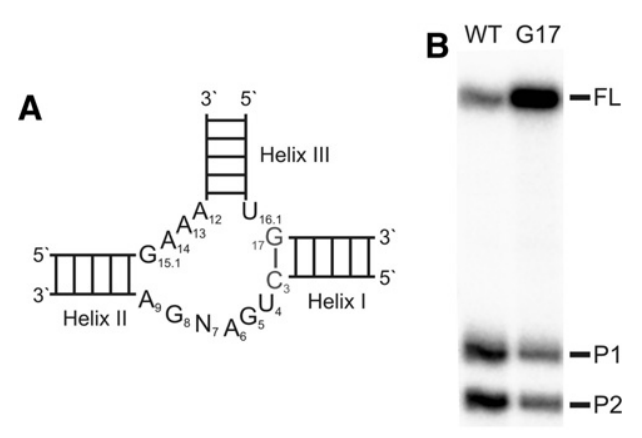

FIGURE 2. G17-modified hammerhead ribozymes. (A) The presence of a guanosine at position 17 (Hertel et al. 1992) is proposed to lead to a trapped conformation that is characterized by a G17-C3 WatsonCrick base pair covering up the cleavage site (Baidya and Uhlenbeck 1997; Kore et al. 1998). (B) Analysis of in vitro transcription reactions of the Xetr5 HHRz wild-type (WT), featuring a C17, and the G17 variant, separated by denaturing polyacrylamide gel electrophoresis. (FL) The full-length RNA; (P1 and P2) the HHRz cleavage products. 
genomic HHRz motifs (Ferbeyre et al. 2000; Rojas et al. 2000; Gräf et al. 2005; Przybilski et al. 2005; De la Peña and GarciaRobles 2010; Jimenez et al. 2011; Perreault et al. 2011; Seehafer et al. 2011). This necessary requirement, however, might not be sufficient, because the identified sequences could adopt alternative folds. In our previous study, we applied thermodynamic selection criteria to discriminate between active $\mathrm{HHRz}$ motifs and those that are inactive, despite fulfilling the descriptor used (Seehafer et al. 2011). With this, we have indentified a series of HHRz motifs from the African claw frog Xenopus tropicalis. To detail the cleavage behavior of these motifs kinetically, we used in vitro transcriptions for the purification of uncleaved full-length RNA. These molecules were subsequently subjected to controlled kinetic analyses. As an internal size marker, we used a G17 variant of the HHRz motif that is strongly impaired in its cleavage reaction, as numerous previous analyses of minimal HHRzs have shown (Ruffner et al. 1990; Shimayama et al. 1995; Zoumadakis and Tabler 1995; Baidya and Uhlenbeck 1997; Baidya et al. 1997; Kore et al. 1998, 2000; Przybilski and Hammann 2007b; Nelson and Uhlenbeck 2008b; Shepotinovskaya and Uhlenbeck 2010). To our surprise, the G17 variant of the X. tropicalis motif Xetr5 (Seehafer et al. 2011) exhibited significant self-cleavage activity under the conditions of in vitro transcription (Fig. 2B). These include the presence of $5 \mathrm{mM} \mathrm{Mg}^{2+}$, under which residual cleavage activity of minimal HHRz can also be observed, while they are inactive at physiological $\mathrm{Mg}^{2+}$ concentrations (De la Peña et al. 2003; Khvorova et al. 2003). Due to the tertiary interactions of helices HI and HII, natural HHRz motifs exhibit cleavage and ligation activity also under the latter conditions.

\section{Kinetic analysis of the cleavage reaction of a G17-containing $\mathrm{HHRz}$}

To analyze whether the G17 mutant of the Xetr5 motif (Fig. $3 \mathrm{~A}$ ) is also cleavage-competent under physiological conditions, we subjected in vitro-transcribed RNA to a controlled cleavage analysis. As can be seen in Figure 3, the G17 variant self-cleaves under these conditions, however, with $k_{\mathrm{obs}}=0.16$ $\min ^{-1}$, about 20 times lower than the wild-type (C17) sequence $\left(k_{\mathrm{obs}}=3.63 \mathrm{~min}^{-1}\right)$ and to a lesser extent (Table 1). To compare the impaired G17 activity with that of mutants with distorted loop interactions, we also generated three variants, in which a consecutive stretch of cytosines replaced the nucleotides of loop 1 (L1pC), loop 2 (L2pC), or both (L1L2pC). As we had shown earlier (Przybilski et al. 2005), the exchange of both loop sequences is particularly detrimental for catalytic activity under physiological conditions. Also
A

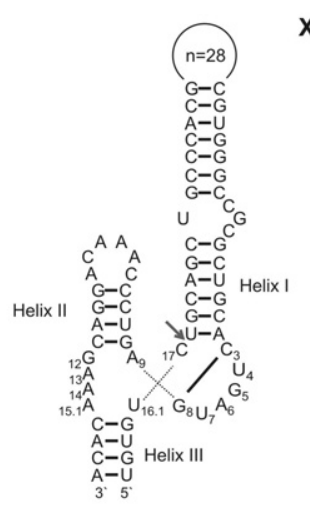

Xetr5 B

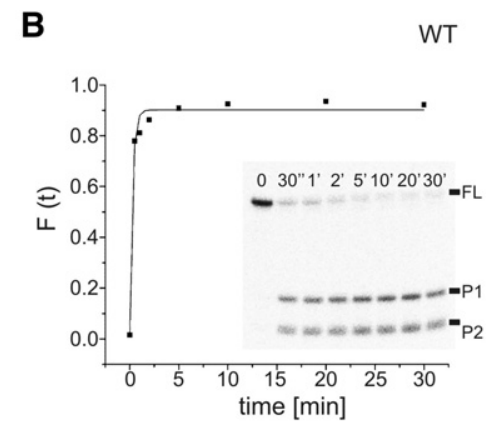

D

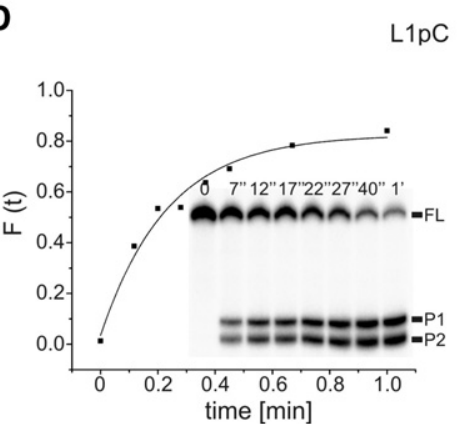

E

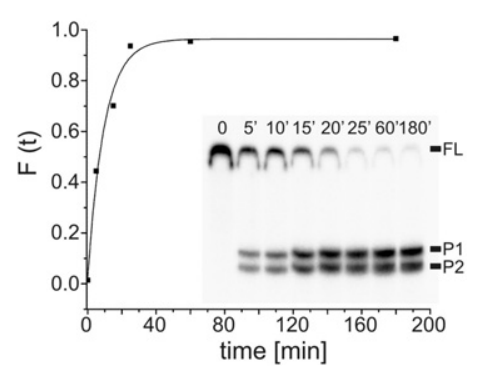

C

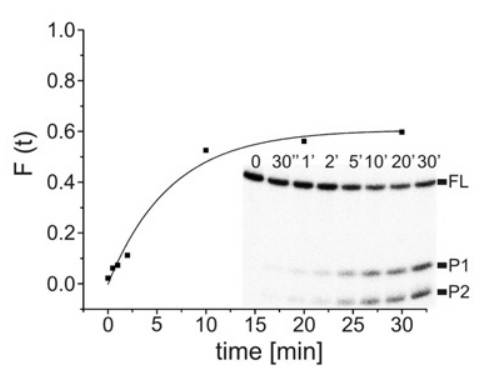

$\mathbf{F}$

L1L2pC

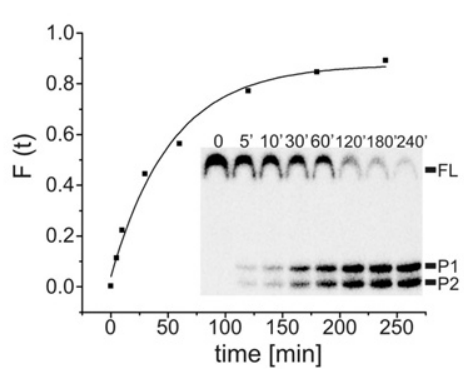

FIGURE 3. The X. tropicalis HHRz Xetr5. (A) Secondary structure of the Xetr5 wild-type (WT) motif. Kinetic analysis of the self-cleavage reactions of the WT $(B)$ and the variants $\mathrm{G} 17(C), \mathrm{L} 1 \mathrm{pC}(D), \mathrm{L} 2 \mathrm{pC}(E)$, and L1L2pC $(F)$. In panels $B-F$, the cleaved fraction $F(t)$ is shown for the indicated time points (in minutes). The solid line represents the fitting function $F_{\text {cleaved }}(t)=F_{0}+F_{\infty} \bullet\left(1-e^{-k \bullet t}\right)$ (Stage-Zimmermann and Uhlenbeck 1998). The insets in panels $B-F$ show the original gel pictures of these analyses with the time points (in minutes) in the top row. Other details are as described in the legend to Figure 2B. 
TABLE 1. Kinetic parameters of the in vitro cleavage reactions of HHRz motifs and variants

\begin{tabular}{llcc}
\hline $\begin{array}{l}\text { HHRz } \\
\text { motif }\end{array}$ & Variant & $\begin{array}{c}\text { Cleavage rate constant } \\
k_{\text {obs }}\left(\mathrm{min}^{-1}\right)\end{array}$ & $\begin{array}{c}\text { Cleavage extent } \\
(\%)\end{array}$ \\
\hline Xetr5 & WT & 3.63 & 90 \\
& G17 & 0.16 & 50 \\
& L1pC & 4.28 & 80 \\
& L2pC & 0.10 & 95 \\
PLMVd & L1L2pC & 0.02 & 80 \\
& WT & 12.62 & 75 \\
& G17 & 0.12 & 50 \\
& L1pC & 4.99 & 80 \\
& L2pC & 6.43 & 90 \\
Ara1 & L1L2pC & 2.92 & 80 \\
& WT & 2.72 & 80 \\
& G17 & 0.14 & 45 \\
& L1pC & 1.11 & 90 \\
& L2pC & 0.09 & 80 \\
Xetr2 & L1L2pC & 0.29 & 90 \\
& WT & 3.42 & 70 \\
& G17 & 0.43 & 20 \\
& L2pC & 0.26 & 80 \\
\hline
\end{tabular}

here, we observe for the Xetr5 L1L2pC variant an $~ 100$-fold decrease in the cleavage rate compared with the wild type, while the extent of cleavage appeared to be similar (Table 1). This indicates that disrupting loop interactions is more detrimental for the cleavage rate than the G17 variant of the Xetr5 sequence, however, at the expense of a reduced cleavage extent.

\section{The tolerance of $\mathrm{G} 17$ varies among different HHRz motifs}

Since sequence variations of core nucleotides are tolerated by individual HHRz motifs differently (Przybilski and Hammann 2007a), we next set out to investigate whether a G17 variant is also active in the background of other HHRz motifs. For this, we performed cleavage analyses on the Arabidopsis thaliana motif Aral (Przybilski et al. 2005), the Peach Latent Mosaic Viroid (PLMVd) (Ambros and Flores 1998) and the X. tropicalis motif Xetr2 (Seehafer et al. 2011). As indicated in Figure 4, each G17 variant displayed cleavage activity, however, with an eightfold to 100 -fold reduced rate as compared with the respective wild-type sequence (Table 1). The cleavage extent for the G17 variants was less variable and leveled to $\sim 50 \%$ of the wild-type sequence (Table 1). As for Xetr5, we also generated the three loop variants $\mathrm{L} 1 \mathrm{pC}, \mathrm{L} 2 \mathrm{pC}$, and $\mathrm{L} 1 \mathrm{~L} 2 \mathrm{pC}$ for the Aral and PLMVd motifs, and only the L1pC loop variant for the Xetr2 motif, since the introduction of the $\mathrm{L} 1 \mathrm{pC}$ sequence in the latter motif led to the formation of an alternative nonHHRz folding (data not shown). These loop variants in general showed a reduced $k_{\text {obs }}$, but an unchanged cleavage extent relative to the wild type (Table 1; the kinetic data are displayed in Supplemental Fig. 1). Compared with the G17 variants, however, there is no clear trend, because loop variants appear to be tolerated better in PLMVd, similarly in Xetr2 and variably in Ara1 (Supplemental Fig. 1; Table 1), in line with earlier observations of idiosyncrasy in the catalytic activity of individual HHRz motifs (Przybilski and Hammann 2007a; Shepotinovskaya and Uhlenbeck 2008).

\section{Ligation activity in G17-containing HHRz}

The most obvious difference in the catalytic cleavage of wildtype and G17 variants was the variability in the $k_{\text {obs }}$ values (Table 1). The PLMVd sequence appeared to be particularly affected by the introduction of the G17 variation. This variation might be due to a different extent by which G17 and wildtype HHRz sequences undergo the reverse ligation reaction. To test this possibility, we have set up ligation reactions, in which we have used the cleavage products isolated after in vitro transcriptions; i.e., both ligation substrates were used in trace amounts. This allows to asses whether religation can occur after self-cleavage, but in view of uncertain concentrations of the two ligation substrates after gel purification, the reaction is refractory to full kinetic analysis. Under these conditions and applying physiological $\mathrm{Mg}^{2+}$ concentrations, we observed slow but significant ligation activity for the G17 variants of all four ribozyme species (Fig. 5). In the three genomically encoded $\mathrm{HHRz}, \mathrm{G} 17$ variants appear to ligate more slowly, or similar to the respective wild-type sequences, yielding a somewhat reduced extent of ligation. The PLMVd HHRz motif showed a deviating ligation activity (Fig. 5B); as for the wild-type sequence, only an extended overnight incubation resulted in a ligation product (data not shown). Its G17 variant displayed initially ligation activity, but the resulting yield was reduced with time (Fig. 5B). Also for other constructs, we occasionally observe upon extended incubation overnight a drop in the ligation extent (data not shown), which might be attributable to RNA degradation.

\section{A $\beta$-galactosidase reporter system reveals HHRz cleavage in vivo}

Although our in vitro kinetic analyses were performed under physiological $\mathrm{Mg}^{2+}$ concentrations, we cannot rule out that the activity of the variant HHRz motifs might differ in living cells. To analyze the in vivo performance of the different motifs, we made use of a recently established $\beta$-galactosidase $(\beta-\mathrm{Gal})$ reporter system in the amoeba Dictyostelium discoideum (Wiegand and Hammann 2013), in which we cloned a HHRz cassette between the stop codon and the poly(A) signal sequences of the lac $Z$ gene (Fig. 6A,B), similar to a reporter described earlier (Khvorova et al. 2003). In case the HHRz does not self-cleave, the mRNA is translated and the $\beta$-Gal activity can be observed in an enzymatic assay using o-nitrophenyl- $\beta$-D-galactopyranoside (ONPG) as substrate. In contrast, no or reduced $\beta$-Gal activity is expected, depending on 
A
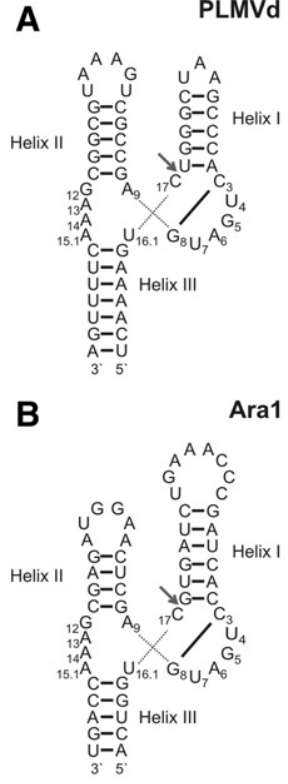

C

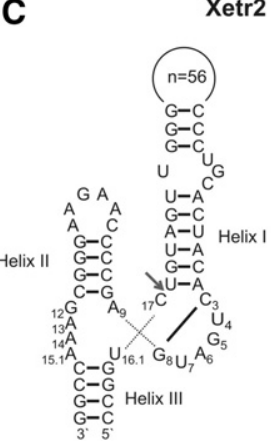

WT

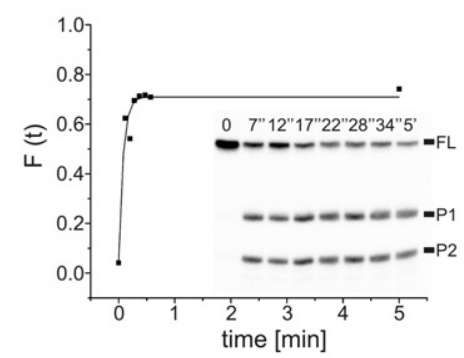

WT

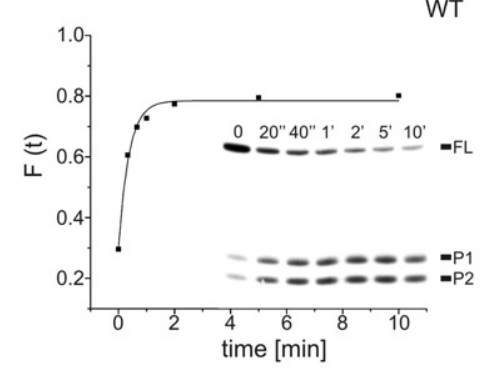

WT

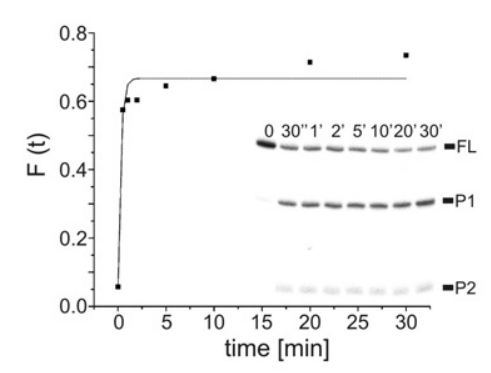

G17

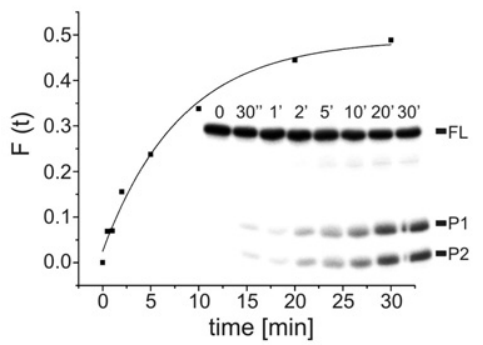

G17

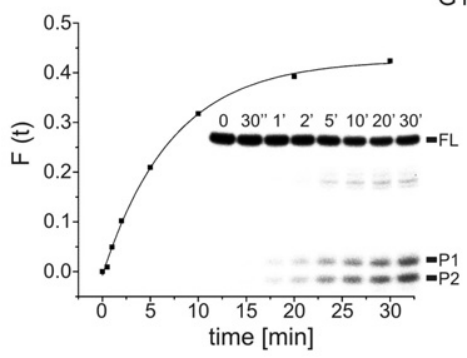

G17

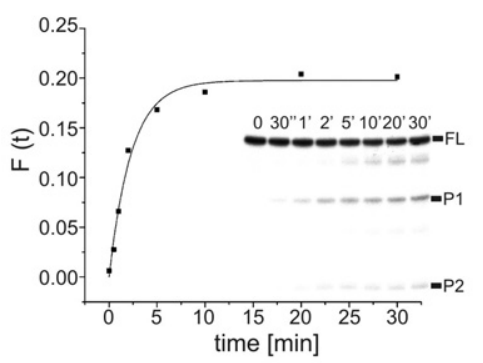

FIGURE 4. Cleavage activity of G17 variants in different HHRz motifs. Shown are the secondary structures (left) and the cleavage analyses for the HHRz WT (middle) and G17 variant (right) for the motifs PLMVd (A), Aral (B), and Xetr2 $(C)$. Other details are as described in the legends to Figures $2 \mathrm{~B}$ and 3 .

the extent of HHRz self-cleavage in vivo. In this reporter system, we have incorporated all HHRz variants that we had tested before in vitro, and analyzed the resulting $\beta$-Gal activity in ONPG assays.

For the analysis of the self-cleavage activity of the variants of the four HHRz motifs used in this study, we transformed D. discoideum AX2 wild-type cells with the $\beta-\mathrm{Gal}-\mathrm{HHRz}$ constructs (Fig. 6A,B) and determined the $\beta$-Gal activity in the resulting strains by ONPG assays. The wild-type sequences of the four ribozymes resulted in no detectable $\beta$-Gal activity, similar to nontransformed $D$. discoideum AX2 cells (negative control), while robust enzymatic activity was observed in strains featuring a construct without $\mathrm{HHRz}$ cassette (positive control) (Fig. 6C-F). The presence of G17-containing $\mathrm{HHRz}$ reduced the $\beta$-Gal activity throughout by $\sim 50 \%$, while changes in the sequences of loops 1 and 2 had variable effects in the different motifs (Fig. 6C-F). This indicates that G17-containing HHRz motifs are active also in vivo and that the activity of loop-modified HHRz depends on the sequence context of the individual motif.

\section{Cleavage activity of G17-containing HHRz in vivo}

Although the data of the $\beta$-Gal reporter point toward ribozyme activity in the respective constructs, we cannot exclude that other parameters, like structural changes in the $\beta-\mathrm{Gal}$ mRNA, influenced the observed changes in enzymatic activity (Fig. 6). We therefore have set out to prove ribozyme cleavage in vivo by monitoring the presence of cleavage products by primer extension assays. For that purpose, we have isolated total RNA from the different $D$. discoideum strains, which we hybridized individually with primers that bind between the HHRz motif and the poly(A) signal (Fig. 6). In case of HHRz-mediated self-cleavage of the mRNA, the primer extension assay is expected to result in a reverse transcript signal between 80 and $200 \mathrm{nt}$ in size, depending on the $\mathrm{HHRz}$ sequence. In the primer extension assay, we observe specific signals of the expected sizes for all variants of either HHRz motif (Fig. 7A-D), but not in the $\beta$-Gal control, which shows a pattern of RT stop signals of variable sizes. In line 
A

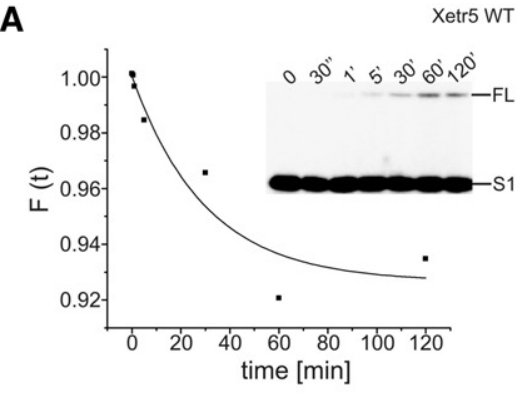

B

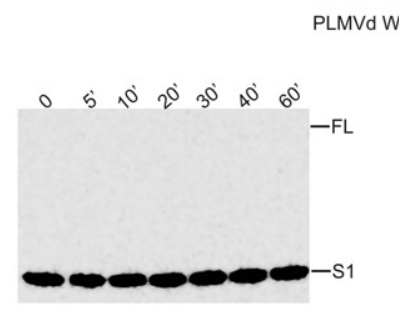

C

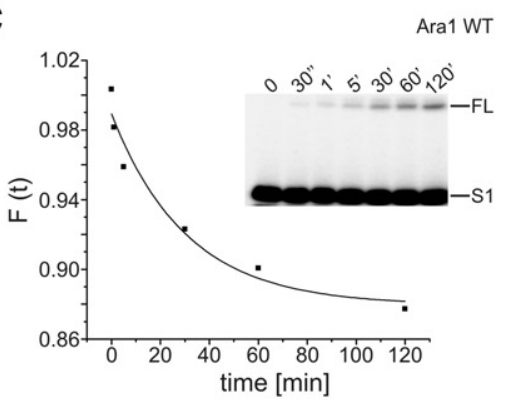

D

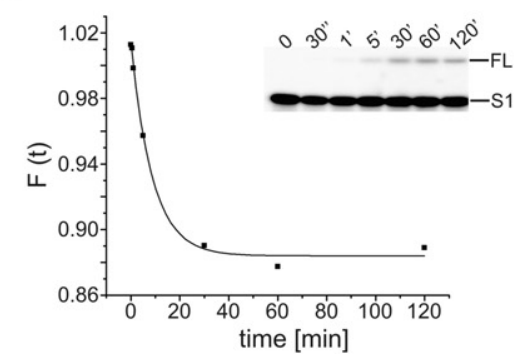

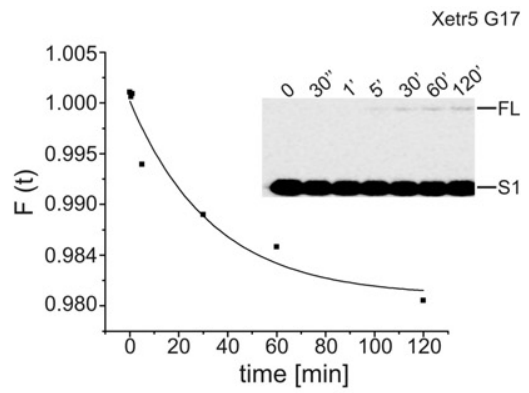
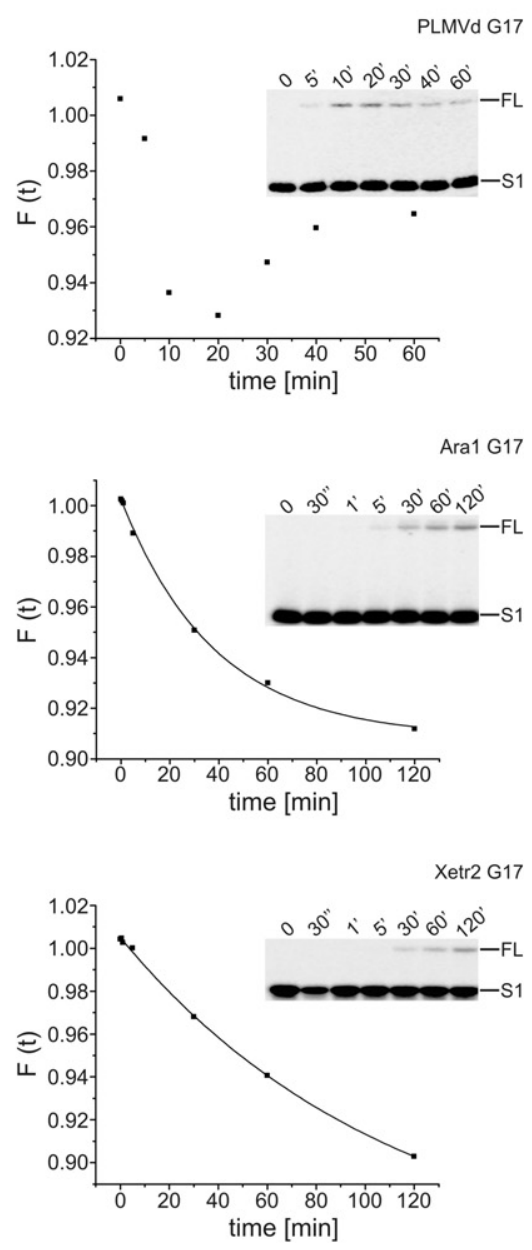

FIGURE 5. Ligation activity of G17 variants in different HHRz motifs. Shown are the ligation analyses for the HHRz WT (left) and G17 variant (right) for the motifs Xetr5 (A), PLMVd (B), Ara1 $(C)$, and Xetr2 $(D)$. Reactions were performed for the indicated times (in minutes) using gel-purified HHRz cleavage products. The cleaved fraction $F(t)$ is plotted against time, as indicated. Other details are as described in the legends to Figures $2 \mathrm{~B}$ and 3.

with both the in vitro cleavage assays (Figs. 3, 4) and the enzymatic activity in the ONPG assay (Fig. 6), we observe bands in the high-molecular-weight range for the $\mathrm{HHRz}$ variants next to the specific signal from the ribozyme cleavage product also. A detailed analysis of these products showed that they have a size similar to that of the uncleaved $\beta$-Gal mRNA, as displayed exemplarily for the Ara1 sequence (Fig. 7E).

\section{DISCUSSION}

The sequence requirements of the catalytic core of the HHRz were established using minimal versions, in which sequence variants were introduced and the kinetic behavior was analyzed in vitro (Ruffner et al. 1990). This resulted in the definition of the standard conserved core nucleotide sequence and the NUH rule for the cleavage site (Shimayama et al. 1995; Zoumadakis and Tabler 1995) that later was extended to become the NHH rule (Kore et al. 1998) since the analysis of tertiary stabilized HHRz has shown that the rules determined with the minimal forms in principle also apply here (Nelson and Uhlenbeck 2008a,b). At the same time, recent data indicated that the core nucleotide requirements might not be as strict as originally anticipated, as summarized in Hammann et al. (2012).

For the minimal form, the G17 variant led to an at least 300 -fold reduction in the cleavage rates in vitro (Ruffner et al. 1990), and a later study showed that a small fraction (4\%) of G17-HHRz can cleave quickly (Nelson and Uhlenbeck 2008b). Our data indicate that this variation in the context of a tertiary stabilized HHRz leads also to a reduction in the cleavage rate. The decrease in this case was, however, only between eightfold and 100-fold for the Xetr2 and the PLMVd HHRz motifs, respectively, while the other two motifs showed an $\sim 20$-fold reduction (Table 1). This indicates that tertiary stabilized HHRz tolerate this variation better than the minimal version, but to varying degrees. All four G17 variants displayed not only a reduced cleavage rate, but also the extent of cleavage went down to $\sim 50 \%$ of that seen in the wildtype HHRz (Table 1). This is in contrast to the kinetic data, which we obtained on the loop nucleotide variants for the four motifs (Table 1; Supplemental Fig. 1), where the extent of cleavage was unaffected while the rates were reduced to varying degrees. Since the loop-loop interactions that were affected by these mutations are responsible for the accelerated cleavage of tertiary stabilized HHRz (Khvorova et al. 2003; Canny et al. 2004; Flores et al. 2004; Penedo et al. 2004; Nelson et al. 2005), the variation in the kinetic rates likely reflects the severity of the disturbance of these interactions in 
A

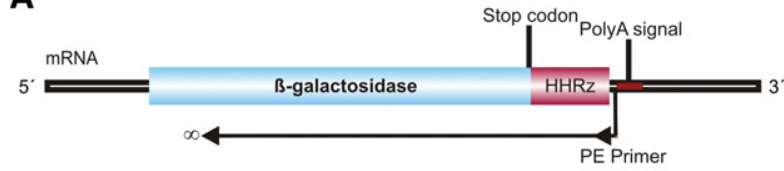

C

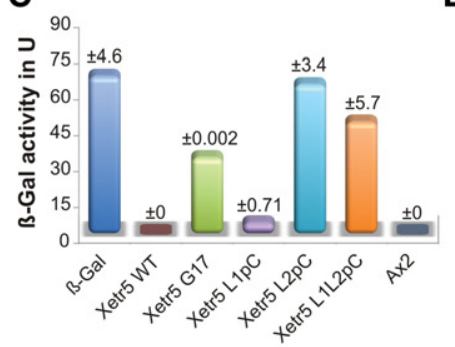

D

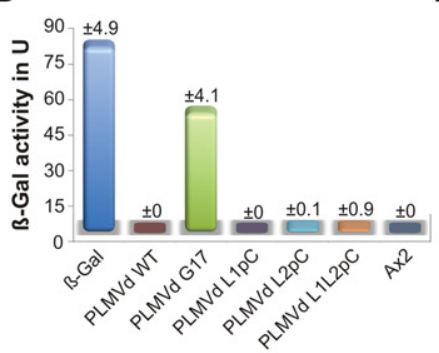

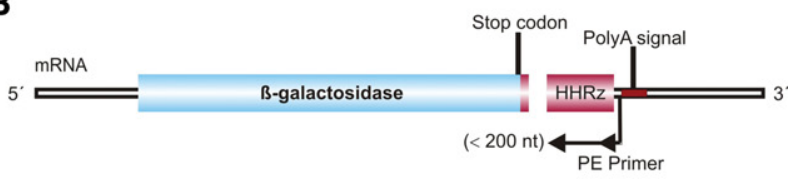

E

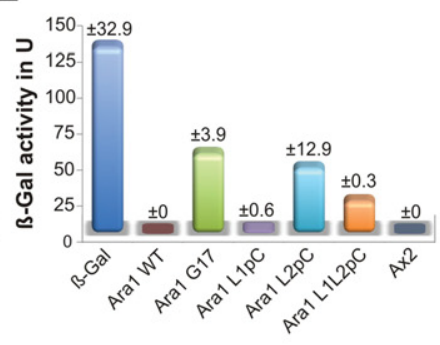

$\mathbf{F}$

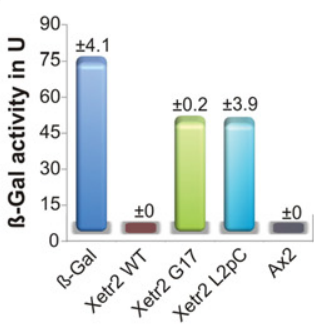

FIGURE 6. The $\beta$-galactosidase reporter system. The HHRz cassette is fused between the stop codon and the poly(A) signal of the $\beta$-galactosidase mRNA. Upon transformation of the reporter in D. discoideum, the mRNA is expected to be stable for a dysfunctional HHRz $(A)$ or destroyed by a functional ribozyme $(B)$. In the latter case, a primer extension (PE) is expected to result in a defined band of $<200$ nucleotides (nt) $(B)$, or, in the former case, in a further extended reverse transcript $(A)$. Enzymatic activity of $\beta$-galactosidase in the reporter systems in $D$. discoideum monitored in ONPG assays for the indicated variants of the HHRz motifs Xetr5 $(C)$, PLMVd $(D)$, Ara1 $(E)$, and Xetr2 $(F)$. The $\beta$-galactosidase activity in U represents the average of three measurements, and the standard deviation is indicated on top of each bar. In each panel, AX2 denotes the wildtype $D$. discoideum strain and $\beta-G a l$ a $D$. discoideum strain that was transformed with a lac $Z$ gene lacking the HHRz cassette.

the individual HHRz motifs. It appears plausible that these variants use the same catalytic strategy as the wild-type HHRz; the reduced loop-loop interactions, however, cause a decrease of the reaction rate, as also concluded from adiabatic morphing calculations (Scott 2007) and the comparison of minimal and tertiary stabilized HHRz (Nelson and Uhlenbeck 2008a). For either G17 variant, the effect appears different, because the same extent of cleavage as in the respective wild type is never reached. This might be explained by a fraction of G17 molecules being trapped in the inactive C3-G17 conformation, as originally proposed for minimal HHRzs (Fig. 2). This interpretation is supported by our obser- vation that a G3-C8 variant is more active in a G17-modified HHRz than in the natural C17 context (data not shown).

Ribozyme-catalyzed ligation can be driven toward the fulllength RNA (Fig. 1B), particularly if one of the substrates is present in elevated concentration, as it is frequently used in pseudo-first-order kinetics. We were interested here in studying whether the ligation also takes place at the equimolar concentrations of substrates that were generated from the cleavage reaction, i.e., whether religation takes place. Among the four tested HHRz, the PLMVd motif and its G17 variant were exceptional in that the G17 variant displayed significantly higher ligation activity (Fig. 5B) with an unusual
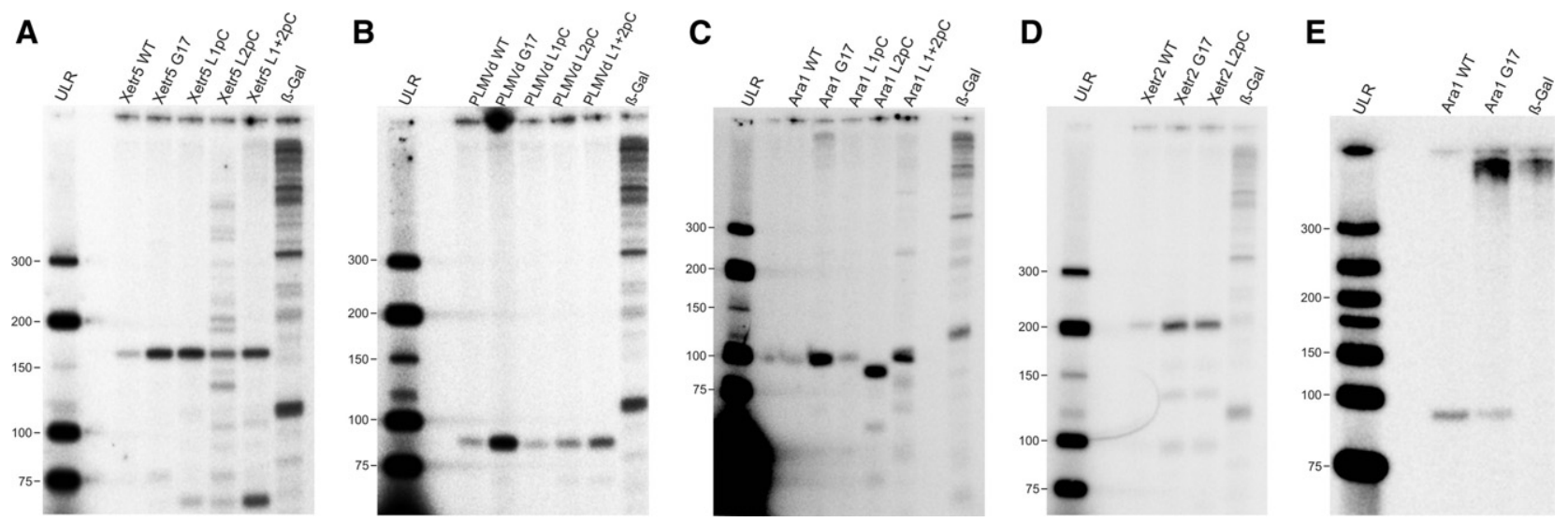

FIGURE 7. Primer extension assays in the $\beta$-Gal reporter system. Products of the reverse transcriptase reaction performed on total RNA from strains with the indicated Xetr5 $(A)$, PLMVd $(B)$, Ara1 $(C)$, and Xetr2 $(D)$ constructs were separated by denaturing polyacrylamide gel electrophoresis. $(E)$ Size comparison of the reaction products from strains with the Aral WT or Aral G17 HHRz construct to that from a strain with an unmodified $\beta$ galactosidase construct. (ULR) Ultra-low-range size marker (Fermentas); sizes are indicated in base pairs to the left of each gel. 
kinetic behavior, while the wild-type sequence was barely active. In contrast to this, we observe ligation activity for all three genomic HHRz motifs and their G17 variants (Fig. 5). The extent of ligation is $\sim 10 \%$ for these three wild-type sequences, and this is in line with their cleavage extent of $\sim 90 \%$ (Table 1 ), using the similar experimental conditions. This indicates that these natural motifs establish an equilibrium between cleavage and ligation. Unlike the extent of cleavage, the three G17 variants display the same extent of ligation as their respective natural motif (Fig. 5). This discrepancy might indicate that the presumed C3-G8 versus C3-G17 alternative does not exist here. A plausible explanation for that might be a preformed C3-G8 base pair in the respective ligation substrate, giving rise to similar ligation rate and extent, independent of whether the second ligation substrate features a $\mathrm{C} 17$ or a G17.

The in vivo data with the $\beta$-Gal reporter system finally are in excellent agreement with the cleavage kinetics determined in vitro. We show in particular that the G17 variant is active also in a physiological context and that the in vitro conditions used are well suited to mimic the cellular activity of the HHRz. This adds G17 to the growing number of HHRz core nucleotide variants with reduced, yet significant, catalytic activity. As discussed earlier, such impaired motifs might be operational in systems where a mechanism is required that can be used as on/off switch (Hammann et al. 2012). The reaction of the PLMVd HHRz, on the other hand, appears to be constitutively on, in line with its function as an RNA endonuclease in processing of multimeric viroid transcripts during rolling circle replication (Branch and Robertson 1984; Tabler and Tsagris 2004; Hammann and Steger 2012). In a new genome database search, we have recently looked for G17-containing HHRz motifs and identified a series of such motifs in several organisms (C Seehafer, A Kalweit, and C Hammann, unpubl.). These motifs were found in organisms that also feature high numbers of wild-type HHRz sequences encoded in their genomes, like Xenopus tropicalis, Schistosoma mansoni, or also Arabidopsis thaliana (Ferbeyre et al. 2000; Rojas et al. 2000; Gräf et al. 2005; Przybilski et al. 2005; De la Peña and Garcia-Robles 2010; Jimenez et al. 2011; Perreault et al. 2011; Seehafer et al. 2011). Future work will address the functionality of such presumed natural G17-containing HHRz motifs.

\section{MATERIALS AND METHODS}

\section{In vitro transcription}

Templates for in vitro transcription were generated synthetically using partially overlapping primers in recursive PCR reactions, as described earlier (Kalweit et al. 2012). The primers of these PCR reactions are listed in Supplemental Table S1 and add XhoI sites at the $5^{\prime}$ and $3^{\prime}$ ends of the fragment for subsequent cloning in expression vector pDneo2a lacZ (Wiegand and Hammann 2013).
The PCR products were cloned in the vector pGEM-T easy and confirmed by sequencing. Transcription reactions were carried out at $37^{\circ} \mathrm{C}$ using T7 RNA polymerase (Milligan et al. 1987) in $40 \mathrm{mM}$ Tris/HCl ( $\mathrm{pH} 8.0$ ), $5 \mathrm{mM} \mathrm{MgCl}_{2}, 2 \mathrm{mM}$ spermidine, $0.01 \%$ Triton X-100. ATP, GTP, and CTP were present in the reaction at a final concentration of $0.5 \mathrm{mM}$ each, and UTP at a final concentration of $0.1 \mathrm{mM}$, supplemented with traces of $\left[\alpha^{32} \mathrm{P}\right] \mathrm{UTP}$. Fulllength transcripts were purified after gel electrophoresis in $10 \%$ or $15 \%$ denaturing polyacrylamide gels containing $7 \mathrm{M}$ urea and visualized by PhosphorImaging. RNA was eluted from gel slices in $40 \%$ formamide, $0.7 \%$ SDS/ $1 \times$ TE by shaking overnight. Eluted RNA was phenolized, ethanol-precipitated, washed in 70\% ethanol, and finally dissolved in $10 \mathrm{mM}$ Tris/ $\mathrm{HCl}$ ( $\mathrm{pH} 7.5$ ) and $25 \mathrm{mM} \mathrm{NaCl}$, supplemented with $0.1 \mathrm{mM}$ EDTA, to prevent self-cleavage.

\section{Kinetic analysis of the in vitro self-cleavage reaction}

The self-cleavage reactions were analyzed as described previously (Kalweit et al. 2012). Kinetic analyses were carried out for each variant at least in duplicate, and deviations in the determined kinetic parameters were below $20 \%$.

\section{Analysis of the in vitro ligation reaction}

For the preparation of the substrates for the ligation reaction, in vitro transcriptions were carried out as described above. The cleavage products were gel-purified and dissolved in reaction buffer $(10 \mathrm{mM}$ Tris/ $\mathrm{HCl}$ at $\mathrm{pH}$ 7.5, $25 \mathrm{mM} \mathrm{NaCl}$, and $0.1 \mathrm{mM}$ EDTA). For the ligation reaction, the radiolabeled substrates were mixed and heated for $2 \mathrm{~min}$ up to $80^{\circ} \mathrm{C}$, followed by snap cooling on ice. The reaction was started by adding $\mathrm{MgCl}_{2}$ to a final concentration of $2 \mathrm{mM}$ and was stopped with denaturing RNA loading dye. Ligation reactions were resolved by electrophoresis on $10 \%$ or $15 \%$ polyacrylamide gels and visualized by PhosphorImaging. Analysis of ligation reactions was carried out as described recently (Kalweit et al. 2012).

\section{Generation of the $\beta-G a l-H H R z$ constructs}

For the generation of the $\beta$-galactosidase-expressing strains with the $\mathrm{HHRz}$ cassette, we used the added XhoI restriction site to excise the HHRz cassette from the cloning vector pGEM-T easy and inserted this construct in the expression vector pDneo2a lacZ (Wiegand and Hammann 2013), which also had been cleaved by XhoI. The localization of the HHRz cassette between the stop codon and the poly (A) signal sequences of the lac $Z$ gene was confirmed by sequencing. The transformation of $D$. discoideum AX2 wild-type cells with the $\beta-\mathrm{Gal}-\mathrm{HHRz}$ constructs was carried out by electroporation as described earlier (Gaudet et al. 2007).

\section{Growth of $D$. discoideum amoebae}

D. discoideum strain AX2 was grown axenically in HL5 medium containing $50 \mu \mathrm{g} / \mathrm{mL}$ ampicillin, $250 \mathrm{ng} / \mathrm{mL}$ amphotericin, $10 \mu \mathrm{g} / \mathrm{mL}$ penicillin, and $10 \mu \mathrm{g} / \mathrm{mL}$ streptomycin at $22^{\circ} \mathrm{C}$ in shaking suspension. In strains transformed with pDneo2a lac $Z$, or derivatives thereof, the medium was supplemented with $10 \mu \mathrm{g} / \mathrm{mL}$ geniticin (G418). 


\section{RNA isolation}

Total RNA was isolated from $2 \times 10^{7}$ cells of axenically grown $D$. discoideum strains using TRIzol (Invitrogen) according to the manufacturer's instructions. The isolated total RNA was resuspended in $\mathrm{dH}_{2} \mathrm{O}$, and the concentration was determined spectrophotomectrically using a NanoDrop (Peqlab).

\section{The $\beta$-galactosidase assay}

For the enzymatic analysis of $\beta$-galactosidase in an o-nitrophenyl- $\beta$ D-galactopyranoside (ONPG) assay (Dingermann et al. 1989), $2 \times$ $10^{6}$ cells were isolated from an exponentially growing shaking culture, centrifuged for $4 \mathrm{~min}$ at $4^{\circ} \mathrm{C}$ at $400 \mathrm{~g}$, and subsequently washed in $2 \mathrm{~mL}$ of Soerensen-phosphate buffer $\left(2 \mathrm{mM} \mathrm{Na}_{2} \mathrm{HPO}_{4}, 15 \mathrm{mM}\right.$ $\mathrm{KH}_{2} \mathrm{PO}_{4}$ at $\mathrm{pH} 6.0$ ). Cells were pelleted again by centrifugation under identical conditions, resuspended in $200 \mu \mathrm{L}$ of ONPG lysis buffer (100 mM sodium phosphate buffer at $\mathrm{pH} 7.0,1 \%$ [w/v] NP-40), and incubated for $10 \mathrm{~min}$ on ice. For the photometric ONPG assay, $5 \mu \mathrm{L}$ of this cell lysate was mixed with $800 \mu \mathrm{L}$ of $\mathrm{Z}$ buffer $(100 \mathrm{mM}$ sodium phosphate buffer at $\mathrm{pH} 7.0,10 \mathrm{mM} \mathrm{KCl}, 1 \mathrm{mM} \mathrm{MgSO}_{4}$, and $7 \mathrm{~mL} / \mathrm{L} \beta$-mercaptoethanol) and $200 \mu \mathrm{L}$ of ONPG solution ( $4 \mathrm{mg} /$ $\mathrm{mL}$ in $100 \mathrm{mM}$ sodium phosphate buffer at $\mathrm{pH}$ 7.0). Absorption measurements were carried out at room temperature at a wavelength of $405 \mathrm{~nm}$. For standardization, the total protein content in the cell lysate was determined in duplicate by Bradford Assays (Bradford 1976), using Roti-Quant (Roth) according to the manufacturer's instructions. For quantitative analysis, the $\beta$-galactosidase activity in the cell lysate was determined according to in which $U$ is the $\beta$-galactosidase activity (in nanomoles per minute per microgram), $\Delta \mathrm{E}_{405}$ the change in absorption (in $\mathrm{min}^{-1}$ ), $\mathrm{V}_{\text {total }}$ the reaction volume (in milliliters), $\mathrm{V}_{\text {protein }}$

$$
\mathrm{U}=\frac{\Delta E_{405} \cdot \mathrm{V}_{\text {total }}}{\mathrm{V}_{\text {protein }} \cdot c_{\text {protein }} \cdot d \cdot \varepsilon}
$$

the volume of the cell lysate in the reaction (in milliliters), $c_{\text {protein }}$ the protein concentration in the cell lysate (in milligrams per milliliter), $\mathrm{d}$ the cuvette path length (in centimeters), and $\varepsilon$ the molar extinction coefficient $\left(4.5 \mathrm{mg} \cdot \mu \mathrm{mol}^{-1} \cdot \mathrm{cm}^{-1}\right)$ (Mount et al. 1996).

\section{Primer extensions assay}

For primer extension, $4-8 \mu \mathrm{g}$ of total RNA was used as template. RNA was denatured for $1 \mathrm{~min}$ at $95^{\circ} \mathrm{C}$ in $50 \mathrm{mM}$ Tris- $\mathrm{HCl}(\mathrm{pH} \mathrm{8.6)}, 60$ $\mathrm{mM} \mathrm{NaCl}$, and $10 \mathrm{mM}$ DTT $(10-\mu \mathrm{L}$ reaction volume) in the presence of a $\left[\gamma^{-32} \mathrm{P}\right]$ ATP radiolabeled primer (PE primer) that was gel-purified before use. Annealing was performed for $4 \mathrm{~min}$ at $54^{\circ} \mathrm{C}$. To this, 5 $\mu \mathrm{L}$ of $5 \mathrm{mMdNTP}$ (each), $5 \mu \mathrm{L}$ of $25 \mathrm{mM} \mathrm{MgCl}_{2}$, and $5 \mu \mathrm{L}$ of Maxima RT (1 unit/ $\mu \mathrm{L}$; Fermentas) in $50 \mathrm{mM}$ Tris- $\mathrm{HCl}(\mathrm{pH} 8.6), 60 \mathrm{mM}$ $\mathrm{NaCl}$, and $10 \mathrm{mM}$ DTT were added. The mixture was incubated for $60 \mathrm{~min}$ at $50^{\circ} \mathrm{C}$, and the reaction was stopped by denaturation for $5 \mathrm{~min}$ at $85^{\circ} \mathrm{C}$. The reaction products were recovered by ethanol precipitation and separated by denaturing gel electrophoresis using polyacrylamide gels at concentrations of $10 \%$ or $15 \%$.

\section{SUPPLEMENTAL MATERIAL}

Supplemental material is available for this article.

\section{ACKNOWLEDGMENTS}

We thank Janis Kruse for her support with the D. discoideum experiments and the members of the Hammann laboratory for discussions. This work was supported by the Deutsche Forschungsgemeinschaft (Heisenberg stipend HA3459/5-2 and grant HA3459/10-1).

Received June 7, 2013; accepted September 6, 2013.

\section{REFERENCES}

Ambros S, Flores R. 1998. In vitro and in vivo self-cleavage of a viroid RNA with a mutation in the hammerhead catalytic pocket. Nucleic Acids Res 26: 1877-1883.

Baidya N, Uhlenbeck OC. 1997. A kinetic and thermodynamic analysis of cleavage site mutations in the hammerhead ribozyme. Biochemistry 36: 1108-1114.

Baidya N, Ammons GE, Matulic-Adamic J, Karpeisky AM, Beigelman L, Uhlenbeck OC. 1997. Functional groups on the cleavage site pyrimidine nucleotide are required for stabilization of the hammerhead transition state. RNA 3: 1135-1142.

Blount KF, Uhlenbeck OC. 2005. The structure-function dilemma of the hammerhead ribozyme. Annu Rev Biophys Biomol Struct 34: 415-440.

Bradford MM. 1976. A rapid and sensitive method for the quantitation of microgram quantities of protein utilizing the principle of proteindye binding. Anal Biochem 72: 248-254.

Branch AD, Robertson HD. 1984. A replication cycle for viroids and other small infectious RNAs. Science 223: 450-455.

Canny MD, Jucker FM, Kellogg E, Khvorova A, Jayasena SD, Pardi A. 2004. Fast cleavage kinetics of a natural hammerhead ribozyme. $J$ Am Chem Soc 126: 10848-10849.

De la Peña M, Garcia-Robles I. 2010. Ubiquitous presence of the hammerhead ribozyme motif along the tree of life. RNA 16: 19431950.

De la Peña M, Gago S, Flores R. 2003. Peripheral regions of natural hammerhead ribozymes greatly increase their self-cleavage activity. EMBO J 22: 5561-5570.

Dingermann T, Reindl N, Werner H, Hildebrandt M, Nellen W, Harwood A, Williams J, Nerke K. 1989. Optimization and in situ detection of Escherichia coli $\beta$-galactosidase gene expression in Dictyostelium discoideum. Gene 85: 353-362.

Dufour D, de la Pena M, Gago S, Flores R, Gallego J. 2009. Structurefunction analysis of the ribozymes of chrysanthemum chlorotic mottle viroid: A loop-loop interaction motif conserved in most natural hammerheads. Nucleic Acids Res 37: 368-381.

Ferbeyre G, Bourdeau V, Pageau M, Miramontes P, Cedergren R. 2000. Distribution of hammerhead and hammerhead-like RNA motifs through the GenBank. Genome Res 10: 1011-1019.

Flores R, Delgado S, Gas ME, Carbonell A, Molina D, Gago S, De la Pena M. 2004. Viroids: The minimal non-coding RNAs with autonomous replication. FEBS Lett 567: 42-48.

Gaudet P, Pilcher KE, Fey P, Chisholm RL. 2007. Transformation of Dictyostelium discoideum with plasmid DNA. Nat Protoc 2: 13171324.

Gräf S, Przybilski R, Steger G, Hammann C. 2005. A database search for hammerhead ribozyme motifs. Biochem Soc Trans 33: 477478.

Hammann C, Lilley DM. 2002. Folding and activity of the hammerhead ribozyme. ChembioChem 3: 690-700.

Hammann C, Steger G. 2012. Viroid-specific small RNA in plant disease. RNA Biol 9: 809-819.

Hammann C, Luptak A, Perreault J, de la Pena M. 2012. The ubiquitous hammerhead ribozyme. RNA 18: 871-885.

Hertel KJ, Pardi A, Uhlenbeck OC, Koizumi M, Ohtsuka E, Uesugi S, Cedergren R, Eckstein F, Gerlach WL, Hodgson R, et al. 1992. Numbering system for the hammerhead. Nucleic Acids Res 20: 3252. 
Hutchins CJ, Rathjen PD, Forster AC, Symons RH. 1986. Self-cleavage of plus and minus RNA transcripts of avocado sunblotch viroid. Nucleic Acids Res 14: 3627-3640.

Jimenez RM, Delwart E, Luptak A. 2011. Structure-based search reveals hammerhead ribozymes in the human microbiome. J Biol Chem 286: 7737-7743.

Kalweit A, Przybilski R, Seehafer C, de la Pena M, Hammann C. 2012. Characterization of hammerhead ribozyme reactions. Methods Mol Biol 848: 5-20.

Khvorova A, Lescoute A, Westhof E, Jayasena SD. 2003. Sequence elements outside the hammerhead ribozyme catalytic core enable intracellular activity. Nat Struct Biol 10: 708-712.

Koizumi M, Ohtsuka E. 1991. Effects of phosphorothioate and 2-amino groups in hammerhead ribozymes on cleavage rates and $\mathrm{Mg}^{2+}$ binding. Biochemistry 30: 5145-5150.

Kore AR, Vaish NK, Kutzke U, Eckstein F. 1998. Sequence specificity of the hammerhead ribozyme revisited; the NHH rule. Nucleic Acids Res 26: 4116-4120.

Kore AR, Carola C, Eckstein F. 2000. Attempts to obtain more efficient GAC-cleaving hammerhead ribozymes by in vitro selection. Bioorg Med Chem 8: 1767-1771.

Martick M, Scott WG. 2006. Tertiary contacts distant from the active site prime a ribozyme for catalysis. Cell 126: 309-320.

Martick M, Horan LH, Noller HF, Scott WG. 2008. A discontinuous hammerhead ribozyme embedded in a mammalian messenger RNA. Nature 454: 899-902.

Milligan JF, Groebe DR, Witherall GW, Uhlenbeck OC. 1987. Oligoribonucleotide synthesis using T7 RNA polymerase and synthetic DNA templates. Nucleic Acids Res 15: 8783-8798.

Mount RC, Jordan BE, Hadfield C. 1996. Reporter gene systems for assaying gene expression in yeast. Methods Mol Biol 53: 239-248.

Murray JB, Scott WG. 2000. Does a single metal ion bridge the A-9 and scissile phosphate groups in the catalytically active hammerhead ribozyme structure? J Mol Biol 296: 33-41.

Murray JB, Terwey DP, Maloney L, Karpeisky A, Usman N, Beigelman L, Scott WG. 1998. The structural basis of hammerhead ribozyme self-cleavage. Cell 92: 665-673.

Nelson JA, Uhlenbeck OC. 2008a. Hammerhead redux: Does the new structure fit the old biochemical data? RNA 14: 605-615.

Nelson JA, Uhlenbeck OC. 2008b. Minimal and extended hammerheads utilize a similar dynamic reaction mechanism for catalysis. RNA 14: $43-54$.

Nelson JA, Shepotinovskaya I, Uhlenbeck OC. 2005. Hammerheads derived from sTRSV show enhanced cleavage and ligation rate constants. Biochemistry 44: 14577-14585.

Penedo JC, Wilson TJ, Jayasena SD, Khvorova A, Lilley DM. 2004. Folding of the natural hammerhead ribozyme is enhanced by interaction of auxiliary elements. RNA 10: 880-888.

Perreault J, Weinberg Z, Roth A, Popescu O, Chartrand P, Ferbeyre G, Breaker RR. 2011. Identification of hammerhead ribozymes in all domains of life reveals novel structural variations. PLoS Comput Biol 7: e1002031.

Prody GA, Bakos JT, Buzayan JM, Schneider IR, Bruening G. 1986. Autolytic processing of dimeric plant virus satellite RNA. Science 231: $1577-1580$.

Przybilski R, Hammann C. 2006. The hammerhead ribozyme structure brought in line. ChembioChem 7: 1641-1644.
Przybilski R, Hammann C. 2007a. Idiosyncratic cleavage and ligation activity of individual hammerhead ribozymes and core sequence variants thereof. Biol Chem 388: 737-741.

Przybilski R, Hammann C. 2007b. The tolerance to exchanges of the Watson/Crick basepair in the hammerhead ribozyme core is determined by surrounding elements. RNA 13: 1625-1630.

Przybilski R, Gräf S, Lescoute A, Nellen W, Westhof E, Steger G, Hammann C. 2005. Functional hammerhead ribozymes naturally encoded in the genome of Arabidopsis thaliana. Plant Cell 17: $1877-1885$

Rojas AA, Vazquez-Tello A, Ferbeyre G, Venanzetti F, Bachmann L, Paquin B, Sbordoni V, Cedergren R. 2000. Hammerhead-mediated processing of satellite pDo500 family transcripts from Dolichopoda cave crickets. Nucleic Acids Res 28: 4037-4043.

Ruffner DE, Stormo GD, Uhlenbeck OC. 1990. Sequence requirements of the hammerhead RNA self-cleavage reaction. Biochemistry 29: 10695-10702.

Scott WG. 2007. Morphing the minimal and full-length hammerhead ribozymes: Implications for the cleavage mechanism. Biol Chem 388: 727-735.

Scott WG, Murray JB, Arnold JRP, Stoddard BL, Klug A. 1996. Capturing the structure of a catalytic RNA intermediate: The hammerhead ribozyme. Science 274: 2065-2069.

Seehafer C, Kalweit A, Steger G, Gräf S, Hammann C. 2011. From alpaca to zebrafish: Hammerhead ribozymes wherever you look. RNA 17: 21-26.

Shepotinovskaya IV, Uhlenbeck OC. 2008. Catalytic diversity of extended hammerhead ribozymes. Biochemistry 47: 7034-7042.

Shepotinovskaya I, Uhlenbeck OC. 2010. Enhanced product stability in the hammerhead ribozyme. Biochemistry 49: 4494-4500.

Shimayama T, Nishikawa S, Taira K. 1995. Generality of the NUX rule: Kinetic analysis of the results of systematic mutations in the trinucleotide at the cleavage site of hammerhead ribozymes. Biochemistry 34: 3649-3654.

Slim G, Gait MJ. 1991. Configurationally defined phosphorothioatecontaining oligoribonucleotides in the study of the mechanism of cleavage of hammerhead ribozymes. Nucleic Acids Res 19: 1183-1188.

Stage-Zimmermann TK, Uhlenbeck OC. 1998. Hammerhead ribozyme kinetics. RNA 4: 875-889.

Tabler M, Tsagris M. 2004. Viroids: Petite RNA pathogens with distinguished talents. Trends Plant Sci 9: 339-348.

Uhlenbeck OC. 1987. A small catalytic oligoribonucleotide. Nature 328: 596-600.

van Tol H, Buzayan JM, Bruening G. 1991. Evidence for spontaneous circle formation in the replication of the satellite RNA of tobacco ringspot virus. J Virol 280: 23-30.

Wang S, Karbstein K, Peracchi A, Beigelman L, Herschlag D. 1999. Identification of the hammerhead ribozyme metal ion binding site responsible for rescue of the deleterious effect of a cleavage site phosphorothioate. Biochemistry 38: 14363-14378.

Wiegand S, Hammann C. 2013. The $5^{\prime}$ spreading of small RNAs in Dictyostelium discoideum depends on the RNA-dependent RNA polymerase RrpC and on the dicer-related nuclease DrnB. PLoS ONE 8: e64804.

Zoumadakis M, Tabler M. 1995. Comparative analysis of cleavage rates after systematic permutation of the NUX consensus target motif for hammerhead ribozymes. Nucleic Acids Res 23: 1192-1196. 

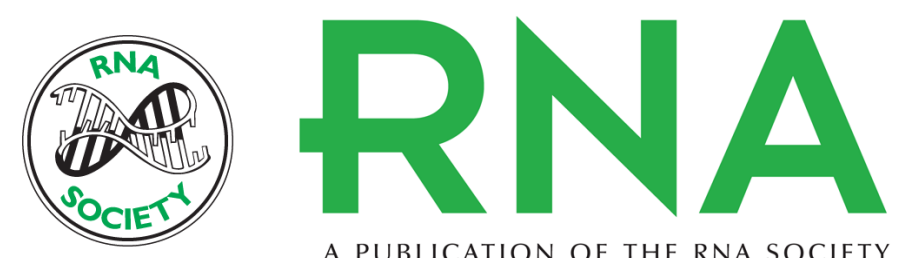

A PUBLICATION OF THE RNA SOCIETY

\section{G17-modified hammerhead ribozymes are active in vitro and in vivo}

Anne Kalweit and Christian Hammann

RNA 2013 19: 1595-1604 originally published online October 21, 2013

Access the most recent version at doi:10.1261/rna.040543.113

Supplemental Material

References

Creative Commons License

Email Alerting Service
http://rnajournal.cshlp.org/content/suppl/2013/10/03/rna.040543.113.DC1

This article cites 58 articles, 15 of which can be accessed free at: http://rnajournal.cshlp.org/content/19/12/1595.full.html\#ref-list-1

This article is distributed exclusively by the RNA Society for the first 12 months after the full-issue publication date (see http://rnajournal.cshlp.org/site/misc/terms.xhtml). After 12 months, it is available under a Creative Commons License (Attribution-NonCommercial 3.0 Unported), as described at http://creativecommons.org/licenses/by-nc/3.0/.

Receive free email alerts when new articles cite this article - sign up in the box at the top right corner of the article or click here.

To subscribe to RNA go to:

http://rnajournal.cshlp.org/subscriptions 